\title{
Correspondence behavior of classical and quantum dissipative directed transport via thermal noise
}

\author{
Gabriel G. Carlo, ${ }^{*}$ Leonardo Ermann, ${ }^{\dagger}$ Alejandro M. F. Rivas, ${ }^{\ddagger}$ and María E. Spina ${ }^{\S}$ \\ Departamento de Física, CNEA, Libertador 8250, (C1429BNP) Buenos Aires, Argentina
}

(Received 11 February 2016; published 25 April 2016)

\begin{abstract}
We systematically study several classical-quantum correspondence properties of the dissipative modified kicked rotator, a paradigmatic ratchet model. We explore the behavior of the asymptotic currents for finite $\hbar_{\mathrm{eff}}$ values in a wide range of the parameter space. We find that the correspondence between the classical currents with thermal noise providing fluctuations of size $\hbar_{\mathrm{eff}}$ and the quantum ones without it is very good in general with the exception of specific regions. We systematically consider the spectra of the corresponding classical Perron-Frobenius operators and quantum superoperators. By means of an average distance between the classical and quantum sets of eigenvalues we find that the correspondence is unexpectedly quite uniform. This apparent contradiction is solved with the help of the Weyl-Wigner distributions of the equilibrium eigenvectors, which reveal the key role of quantum effects by showing surviving coherences in the asymptotic states.
\end{abstract}

DOI: 10.1103/PhysRevE.93.042133

\section{INTRODUCTION}

A pioneering paper by Feynman [1] reignited interest in directed transport [2-4], which means the unbiased transport phenomena in systems which are driven out of equilibrium. There is a wealth of fields for their application such as biology [5], nanotechnology [6], chemistry [7], cold atoms [8,9], and Bose-Einstein condensates [10-13]. Among all these alternatives, we focus in deterministic ratchets with dissipation generally associated with a classical asymmetric chaotic attractor $[14,15]$; the quantum versions lead to interesting applications in cold atoms [16]. The classical aspects of the parameter space of this system have been studied in detail in [17]. These new results revealed that the isoperiodic stable structures (ISSs, Lyapunov stable islands) have a fundamental role in the current shape. The quantum counterparts of these structures (QISSs) [18,19] have proven to be very well approximated by means of a thermal coarse graining of the classical dynamical equations (i.e., adding thermal noise of the order of $\hbar_{\mathrm{eff}}$ ) in representative cases. Taking into account these examples, it was recently found [20] that the PerronFrobenius operators associated with the classical evolution with thermal noise and the quantum superoperators without it show very similar spectra. It deserves noticing that the study of Perron-Frobenius operators in the Ulam approximation is very useful in open systems theory [21].

In a recent publication a semiclassical approach was taken into account [22]. There it was argued that an effective (semi)classical map with noise could be used as a direct replacement for the quantum system itself and several consequences have been derived. We take into account a thermal-like noise, i.e., a Gaussian noise whose strength is given by $\hbar_{\mathrm{eff}}$, leaving no free parameters. It is important to underline that the effective temperature to which it can be associated is different throughout the parameter space. Nevertheless, our thermal noise approach and the semiclassical one are very

\footnotetext{
*carlo@tandar.cnea.gov.ar

†ermann@tandar.cnea.gov.ar

†rivas@tandar.cnea.gov.ar

§spina@tandar.cnea.gov.ar
}

much alike. In this work we verify our conjecture about the role of the coarse graining due to quantization: it shares the main properties of a thermal coarse graining for this kind of dissipative system. In fact, it induces chaotic behavior in situations where one expects a simple attractor for instance [18]. Thus, it is of general nature and is applicable to any finite $\hbar_{\text {eff }}$ value, including the semiclassical regime which of course is compatible with it. Finally, the quantum regime is not only interesting from a theoretical point of view, but also for a great number of experimental situations in which the semiclassical limit is not reached.

However, there are relevant finite $\hbar_{\text {eff }}$ quantum effects that cannot be reproduced by a classical map. In this paper we find that though the general correspondence is very good, there are specific regions in which this mechanism shows limitations. This is clearly seen with the help of the main quantity of interest in directed transport, i.e., the asymptotic current, keeping in mind that it is just an average quantity that does not reflect a complete picture. Also, it is important to notice that transitory regimes are interesting but they are not the main objective here. But, when we study the spectra of the Perron-Frobenius operators with thermal noise and the quantum superoperators without it we find an almost uniform correspondence, with no clear signs of these discrepancy regions. This could be puzzling in view of the current behavior previously mentioned. Moreover, we already know [18] that chaotic limit sets provide quite a bit of mixing with no need of noise, making uniformity unexpected. On the other hand, even for the chaotic case the thermal coarse graining is necessary in order to make both spectra agree [20]. To clarify this we explore the morphology of the eigenvectors associated with the equilibrium eigenvalues. Here we find that surviving coherences (which cannot be reproduced by means of a classical model with noise) are a key indicator to understanding the limits that this mechanism shows in describing some asymptotic currents. This also provides valuable information needed to develop a semiclassical approximation of the equilibrium eigenstates - a long standing objective-perhaps taking into account convenient wave packets and the classical dynamics with thermal noise.

This paper has the following structure: In Sec. II we introduce our model which is a modified kicked rotator with 
dissipation, clearly explaining the way in which we add the thermal noise in the classical model to find the correspondence with the quantum one. In Sec. III we explore the difference between the classical and quantum asymptotic currents in the parameter space, finding that there are regions in which the correspondence is not so accurate. In Sec. IV we systematically explore the spectra of the classical PerronFrobenius operator with thermal noise and the quantum superoperator in the parameter space, which show a seemingly uniform correspondence. In Sec. V we explain this apparent contradiction by extending this study to the phase space and using the Weyl-Wigner distributions of the equilibrium eigenvectors. In Sec. VI, we present our conclusions.

\section{MODEL AND CALCULATION METHODS}

We consider a particle moving in one dimension $[x \in$ $(-\infty,+\infty)$ ] periodically kicked by the asymmetric potential:

$$
V(x, t)=k\left[\cos (x)+\frac{a}{2} \cos (2 x+\phi)\right] \sum_{m=-\infty}^{+\infty} \delta(t-m \tau),
$$

where $k$ is the strength of each kick and $\tau$ is the kicking period. When adding dissipation we obtain a dissipative ratchet system that can be written as the following map [16,17]:

$$
\begin{aligned}
& \bar{n}=\gamma n+k[\sin (x)+a \sin (2 x+\phi)], \\
& \bar{x}=x+\tau \bar{n} .
\end{aligned}
$$

Here $n$ is the momentum variable conjugated to $x$ and $\gamma$ $(0 \leqslant \gamma \leqslant 1)$ is the dissipation parameter. The conservative limit is reached at $\gamma=1$, whereas the value $\gamma=0$ gives the maximum damping. In order to simplify the parametric dependence it is usual to introduce a rescaled momentum variable $p=\tau n$ and the quantity $K=k \tau$. The directed current emerges as a consequence of breaking the spatial and temporal symmetries by adopting $a \neq 0$ with $\phi \neq m \pi$, and $\gamma \neq 1$, respectively. It is worth mentioning that we take $a=0.5$ and $\phi=\pi / 2$ throughout this work.

We have conjectured [18] that the main effects of the quantum fluctuations are similar to those of Gaussian fluctuations of the order of $\hbar_{\mathrm{eff}}$ in the classical analog $\left(\hbar_{\mathrm{eff}}\right.$ is the effective Planck constant to be defined in the next paragraph). In order to introduce them we replace the first line of Eq. (2)_with $\bar{n}=\gamma n+k[\sin (x)+a \sin (2 x+\phi)]+\xi$. Though the essential idea is the Gaussian nature of fluctuations on the effective Planck scale, we can associate the noise variable $\xi$ with a temperature $T$ by means of the relation $\left\langle\xi^{2}\right\rangle=2(1-\gamma) k_{B} T$, where $k_{B}$ is the Boltzmann constant (which we take equal to 1). Moreover, in this work we take $T=\hbar_{\text {eff }} /[2(1-\gamma)]$. Note that we have explicitly fixed the value of $T$ as a function of $\hbar_{\mathrm{eff}}$ and $\gamma$. This leaves no free parameters in order to test the behavior of our conjecture in this situation. However we underline that this is not essential for it to be valid. In the following, when we refer to classical properties or quantities it is assumed that they correspond to the classical system with thermal noise unless otherwise stated.

The corresponding quantum model without thermal noise is given by $x \rightarrow \hat{x}, n \rightarrow \hat{n}=-i(d / d x)(\hbar=1)$. Since $[\hat{x}, \hat{p}]=$ $i \tau$ (where $\hat{p}=\tau \hat{n}$ ), the effective Planck constant is $\hbar_{\mathrm{eff}}=\tau$. The classical limit corresponds to $\hbar_{\text {eff }} \rightarrow 0$, while $K=\hbar_{\text {eff }} k$ remains constant. We fix $\hbar_{\mathrm{eff}}=0.137$ in this work since we are not interested in reaching the classical limit. Dissipation at the quantum level is introduced by means of the master equation [23] for the density operator $\hat{\rho}$ of the system

$\dot{\hat{\rho}}=-i\left[\hat{H}_{s}, \hat{\rho}\right]-\frac{1}{2} \sum_{\mu=1}^{2}\left\{\hat{L}_{\mu}^{\dagger} \hat{L}_{\mu}, \hat{\rho}\right\}+\sum_{\mu=1}^{2} \hat{L}_{\mu} \hat{\rho} \hat{L}_{\mu}^{\dagger} \equiv \Lambda \rho$.

Here $\hat{H}_{s}=\hat{n}^{2} / 2+V(\hat{x}, t)$ is the system Hamiltonian, $\{$,$\} is$ the anticommutator, and $\hat{L}_{\mu}$ are the Lindblad operators given by $[24,25]$

$$
\begin{aligned}
& \hat{L}_{1}=g \sum_{n} \sqrt{n+1}|n\rangle\langle n+1|, \\
& \hat{L}_{2}=g \sum_{n} \sqrt{n+1}|-n\rangle\langle-n-1|,
\end{aligned}
$$

with $n=0,1, \ldots$ and $g=\sqrt{-\ln \gamma}$ (due to the Ehrenfest theorem).

All this is enough to compare the behavior of the asymptotic currents. But if we want to go further in a systematic study of the classical to quantum correspondence we need to incorporate more quantities. The classical densities in phase space evolve with the Perron-Frobenius operator arising from the Liouville equation corresponding to the map in Eq. (2). A discretization of phase space gives rise to the Ulam method [26], which is a coarse-grained approximation to the Perron-Frobenius operator [27,28]. The procedure consists of defining the Ulam matrix $S$ by means of dividing the phase space into $M^{2}$ cells and propagating $n_{\text {tr }}$ random points from each cell $j$ with the classical map. The number $n_{i j}$ of trajectories arriving to cell $i$ from the cell $j$ allows us to write the elements of $S$ as $S_{i j}=\frac{n_{i j}}{n_{\text {tr }}}$. It is important to notice that this discretization is comparable to a diffusive noise of order $h_{\mathrm{eff}}^{\mathrm{PF}} \propto \frac{1}{M}$. For homogeneous systems and sufficiently large values of $M$ the Ulam method converges to the spectrum of the continuous system. If the coarse graining of the Ulam method is smaller than that attributable to the thermal fluctuations (i.e., $h_{\text {eff }}^{\mathrm{PF}} \leqslant h_{\mathrm{eff}}$ ) the results obtained are independent of $h_{\mathrm{eff}}^{\mathrm{PF}}$. In the following, the expression Perron-Frobenius operator will make reference to its Ulam approximation.

In the quantum case the evolution of the density matrix is given by $\rho_{t+1}=e^{\Lambda} \rho_{t}$, where $e^{\Lambda}$ is a non-unital superoperator of dimension $N^{2} \times N^{2}$ constructed by numerical integration of Eq. (3). Here $h_{\text {eff }} \propto \frac{1}{N}$. It is important to notice that thanks to dissipation the classical and quantum distributions are bounded in the momentum coordinate. This bound provides us with a natural criterion for the truncation of the phase space, making sure to take into account all the relevant dynamics for the values of the parameters that we have considered. This translates directly into finite (super)operators. Finally, for the diagonalization of $S$ and $e^{\Lambda}$ we have used the Arnoldi method [28,29].

\section{CLASSICAL AND QUANTUM ASYMPTOTIC CURRENTS}

We first look at the properties of asymptotic ratchet currents $J=\langle p\rangle$ (where \langle\rangle stands for either the classical or quantum averages). These averages are done over classical 

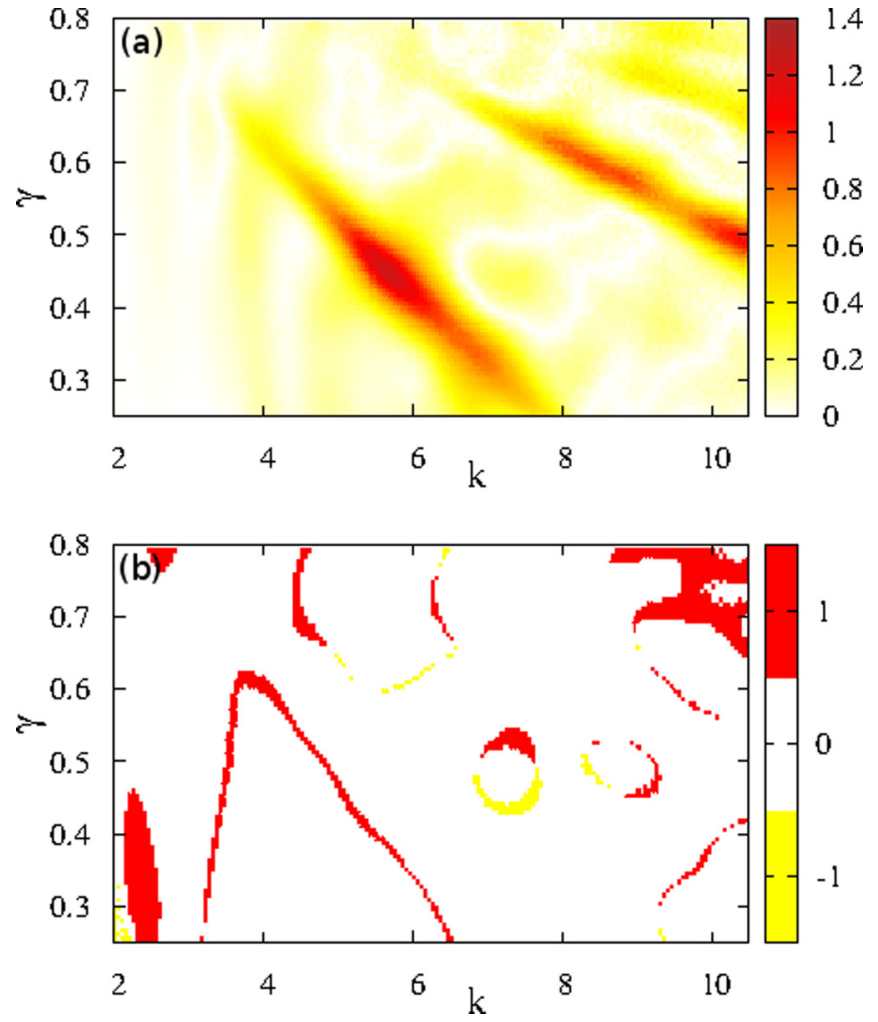

FIG. 1. (a) We display the distance between the classical and quantum asymptotic currents given by $\left|J_{c}^{\text {th }}-J_{\mathrm{q}}\right|$. We have considered this in order to better reflect the discrepancy in the approximation. (b) We show the sign difference $\left[\operatorname{sgn}\left(J_{\mathrm{c}}^{\text {th }}\right)-\operatorname{sgn}\left(J_{\mathrm{q}}\right)\right] / 2$.

random initial conditions having $p \in[-\pi, \pi]$ and $x \in[0,2 \pi]$ $\left(\left\langle p_{0}\right\rangle=0\right)$ in all cases, and also over their quantum density operator counterpart in Hilbert space. They are also taken over time. A word of caution is in order here; this quantity is an average and as such the information it provides regarding classical-quantum correspondence is limited. Nevertheless it is the main quantity of interest for directed transport systems, along with the widths of distributions [19]. In Fig. 1(a) we show the difference $\left|J_{\mathrm{c}}^{\text {th }}-J_{\mathrm{q}}\right|$, where $J_{\mathrm{c}}^{\text {th }}$ stands for the classical current and $J_{\mathrm{q}}$ for the quantum one. This shows the distance between them, i.e., the discrepancy in the approximation. The differences are in general quite small as can be seen from the white-yellow overall appearance of the plot. For this statement to be meaningful one has to keep in mind the much higher currents of the original classical system without thermal noise. Also, one should remember that the width in $p$ of the distributions can be two orders of magnitude greater than these values. This is proof that, regarding currents, our conjecture works very well, despite that we have fixed the size of the fluctuations at exactly $\hbar_{\text {eff }}$, a too restrictive requirement.

But this image also shows that a plain replacement of the quantum currents with this kind of approximation could lead to quantitatively wrong results. This is especially evident in some regions of the isoperiodic structures $B_{1}$ and $B_{2}$ [22]. There are also minor differences in the other $B$ structures and in a few cases of chaotic regions. It is also interesting to note that some of the better correspondence arises in borders of what is left of the original classical parameter space regions associated with either chaotic or regular behavior. These are small fluctuations but still give a hint of a missing ingredient that could be responsible for the enhancement of one or the other structure at either side of this frontier. In the following sections we will explain its nature.

In Fig. 1(b) the sign difference $\left[\operatorname{sgn}\left(J_{\mathrm{c}}^{\text {th }}\right)-\operatorname{sgn}\left(J_{\mathrm{q}}\right)\right] / 2$ is displayed. This shows that for almost all cases the sign is very well reproduced, but also that, though being quite similar, the borders of the positive and negative regions do not necessarily coincide.

\section{SPECTRAL CORRESPONDENCE}

The asymptotic current is the main quantity of interest in directed transport but, being an average, it is not a very precise gauge for measuring the correspondence behavior in which we are interested to test in this work. Then we turn to studying complementary quantities. In this section we look at the properties of the spectra of the Perron-Frobenius operators and the quantum superoperators [20]. For that we define the average distance between the classical and the quantum spectra $\Delta_{\mathrm{q}-\mathrm{c}}$ as the average of the Euclidean distances in the complex plane between the eigenvalues $\lambda$ that are nearest neighbors from both spectra. In Fig. 2 we show this measure for five different values of the dissipation parameter $\gamma=0.4,0.45$, 0.5, 0.55, and 0.6 (from Figs. 2(e) to 2(a), correspondingly), as a function of $k$. We have considered just the longest lived eigenvalues, but in order to check the convergence of this measure we have taken into account three different values for the minimum modulus required to be considered as such $(0.25,0.35$, and 0.4 , by means of green, red, and violet solid lines, respectively). The behavior is quite consistent and these curves show no meaningful jumps, all of them being around one order of magnitude lower than the minimum moduli. This tells us not only that the spectral correspondence is good but also that it is approximately uniform. As a guide to the eye we have also drawn the classical $J_{\mathrm{c}}$ without noise, so as to reflect the insensitivity of this measure to the changes in the nature of the dynamics that takes place in the parameter space. This is an unexpected result a priori, since in Sec. III it became clear that the approximation does not work in a uniform way throughout the parameter space. Moreover, we already knew from our previous work that the chaotic currents are already in good correspondence without needing extra fluctuations. This guarantees nonuniformity, no matter which noise model one chooses.

When we look deeper into the details of this correspondence we see how the spectra follow each other as the parameter $k$ is varied. In Fig. 3(a) we show the distances between the classical and quantum eigenvalues for $\gamma=0.6$ and $k$ between 5 and 10, taking steps $\Delta k=0.05$. In Fig. 3(b) we display a zoom that highlights this remarkably uniform coincidence.

\section{PHASE-SPACE REPRESENTATION OF THE ASYMPTOTIC EIGENSTATES}

What could explain this apparent paradox? We know from a previous work [20] that even for the already similarly behaved chaotic cases one needs to add Gaussian noise in order to recover a quantumlike spectrum. This would put on 


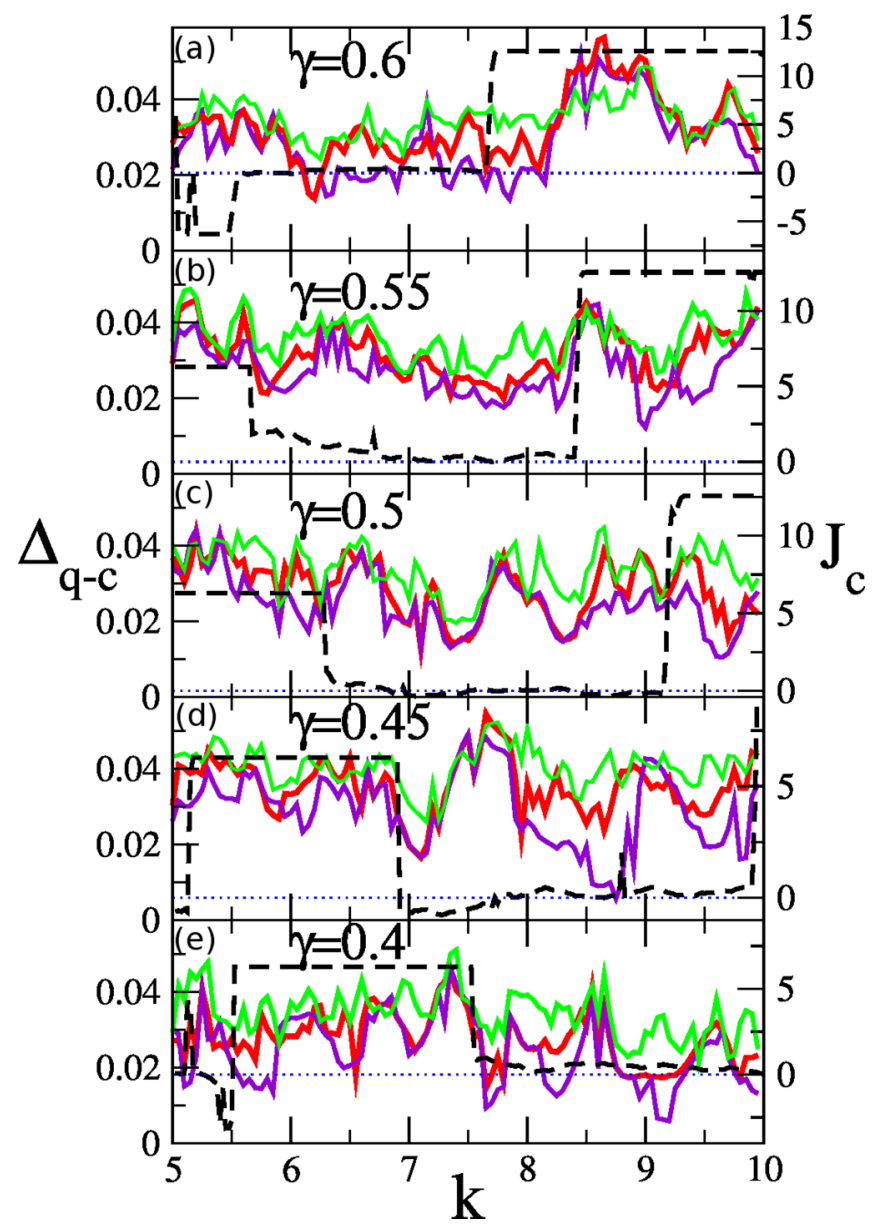

FIG. 2. Average distance between classical and quantum eigenvalues $\lambda$ in complex plane vs $k$ for values of $\gamma=0.4,0.45,0.5,0.55$, and 0.6 from (e) to (a), correspondingly. Green, red, and violet solid lines show the average distance for eigenvalues with moduli larger than $0.25,0.35$ and 0.4, respectively (values are shown in left axis). Dashed black lines represent classical currents (with values shown in right axis) with dotted blue lines showing zero current.

an equal footing the spectra for different regions of parameter space in terms of their classical-quantum correspondence. And one has to keep in mind that all the information needed to obtain the asymptotic currents is contained in the equilibrium eigenstates, which of course have all the same eigenvalue 1. It is important to notice that there is only one equilibrium eigenstate associated with the eigenvalue 1 for each case, i.e., for each selection of the parameter values. So, differences in these states could entirely explain the differences that we have found in Sec. III, differences which are not necessarily reflected in the rest of the eigenvalues as a whole.

On one hand we have the right invariant eigenvectors of the Perron-Frobenius operator $R_{\lambda_{0}}$ which are real and non-negative and can be directly taken as probability distributions in phase space. On the other, we have the Weyl-Wigner symbols for the right invariant eigenvectors of the quantum superoperator $\hat{R}_{\lambda_{0}}$ which can be taken as density matrices satisfying $\operatorname{Tr}\left(\hat{R}_{\lambda_{0}}\right)=1$. The Weyl-Wigner symbols for an $N$-dimensional Hilbert space are defined in a redundant $2 N \times 2 N$ discrete phase space [30]. This is formed by the grid of points $x=\frac{1}{N}(a, b)$ with $a$ and $b$
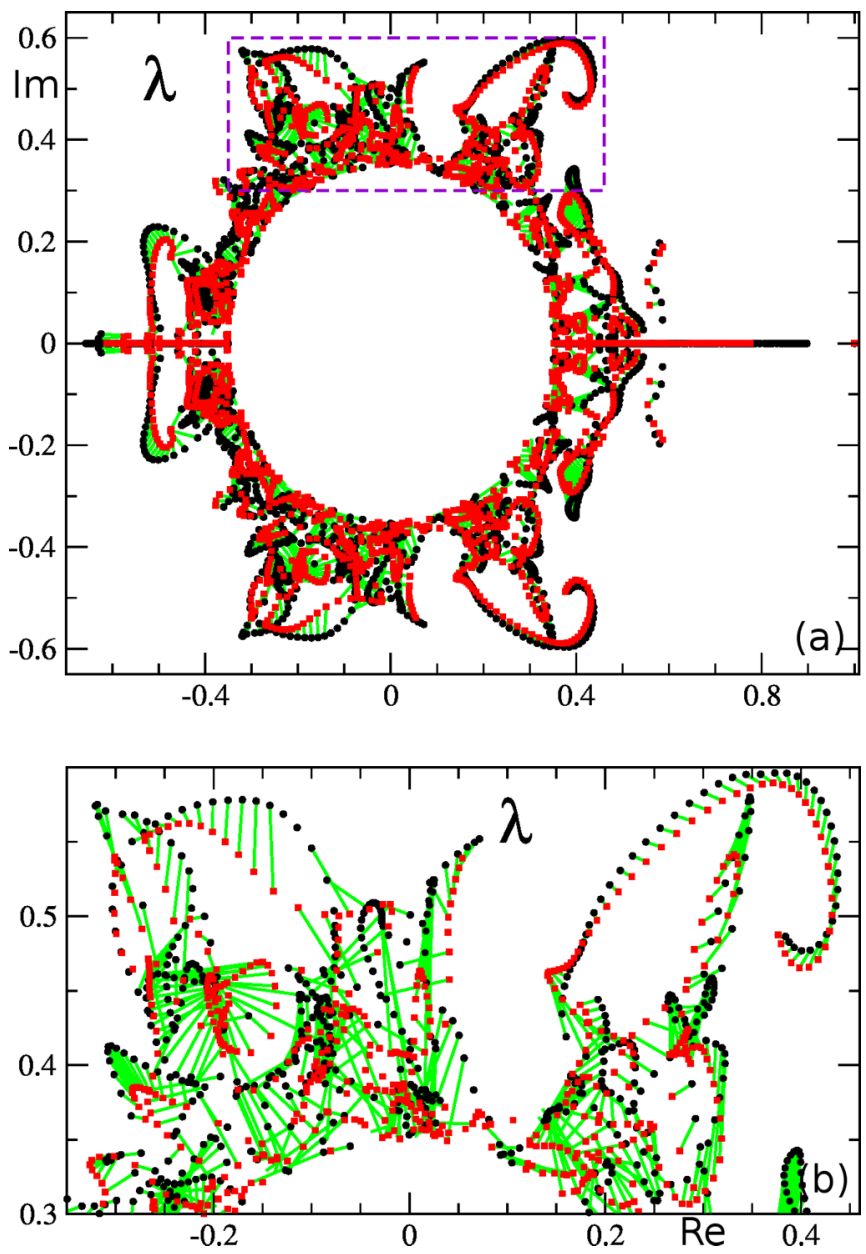

FIG. 3. Black circles and red squares show the eigenvalues $\lambda$ of the classical and quantum systems, respectively, displayed in the complex plane. The green lines represent the distances between them, they are only computed when classical and quantum eigenvalues are larger than 0.35 (in modulus). We have taken $\gamma=0.6$ and $k$ between 5 and 10 (with steps $\Delta k=0.05$ ). The highlighted area (with a dashed violet line) of (a) is shown in (b).

semi-integer numbers running from 0 to $N-1 / 2$. In this way, the Weyl-Wigner symbol $R(x)$ of the operator $\hat{R}$ is obtained from its matrix elements in the coordinate representation as

$$
R(x)=\sum_{n=0}^{N-1}\left\langle q_{2 b-n}|\hat{R}| q_{n}\right\rangle \exp \left(\frac{i 2 \pi}{N} 2 a(b-n)\right) .
$$

In order to get rid of redundancies and "ghost images" derived essentially from the cylindrical topology of our phase space, we use a method that has been developed by Argüelles and Dittrich [31] (for more details on this calculation, see [20]).

A convenient measure to compare both distributions is the overlap. To calculate these overlaps we take into account that any state $\hat{R}$ can be represented by $R(x)$ with $x=(p, q)$ a point in phase space. For the classical states, $R(x)$ stands for the right eigenvector, while for the quantum ones, $R(x)$ is the Weyl-Wigner symbol. Hence, given any two states $\hat{R}_{1}$ and $\hat{R}_{2}$, 

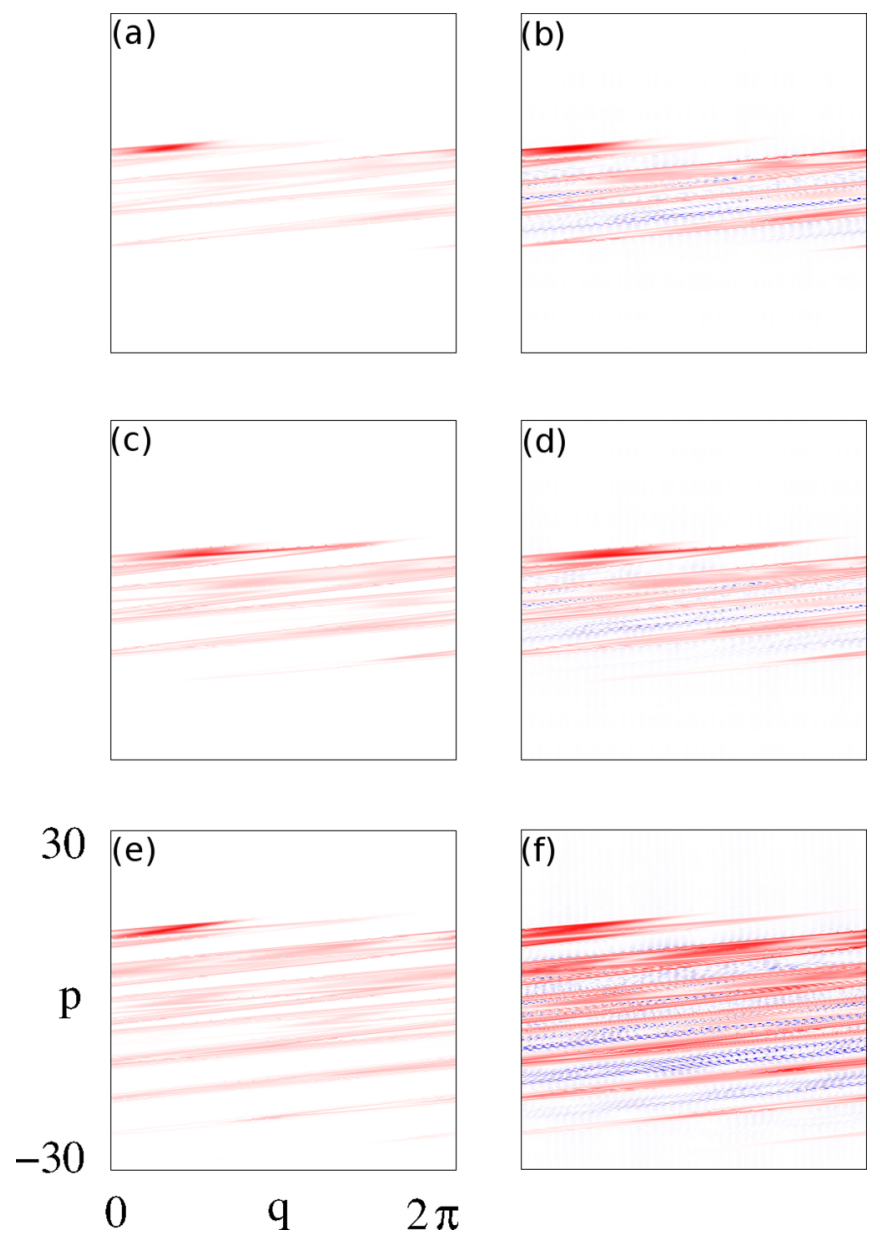

FIG. 4. Three examples of distributions of classical (left column) and quantum (right column) equilibrium states. Panels (a) and (b) correspond to $k=5$ and $\gamma=0.5$, panels (c) and (d) to $k=5.55$ and $\gamma=0.55$, and panels (e) and (f) to $k=9.25$ and $\gamma=0.55$. Negative values are highlighted in blue (gray) while red (darker shades of gray) stand for the positive ones. The scale is fixed in each panel, taking into account the maximum of the corresponding distribution.

their overlap is defined as

$$
\begin{aligned}
O & \left(\hat{R}_{1}, \hat{R}_{2}\right) \\
& =\operatorname{Tr}\left(\hat{R}_{1} \hat{R}_{2}\right) / \sqrt{\left[\operatorname{Tr}\left(\hat{R}_{1}^{2}\right) \operatorname{Tr}\left(\hat{R}_{2}^{2}\right)\right]} \\
& =\sum_{x} R_{1}(x) R_{2}^{*}(x) / \sqrt{\left[\left(\sum_{x}\left|R_{1}(x)\right|^{2}\right)\left(\sum_{x}\left|R_{2}(x)\right|^{2}\right)\right]},
\end{aligned}
$$

where $R^{*}(x)$ and $|R(x)|$ stand respectively for the complex conjugate and absolute value of $R(x)$. The overlap defined above is a complex magnitude, its modulus is invariant even though its argument depends on the relative phase between the eigenvectors. Also, when this relative phase is null, $O\left(\hat{R}_{1}, \hat{R}_{2}\right)$ is real.

We will focus our attention on the greatest discrepancy regions $B_{1}$ and $B_{2}$, which explain almost all the main classicalquantum differences. In Fig. 4 we show three examples, two of them where the discrepancy in the classical approximation is maximal (on the order of 1 in the current), and one in which the
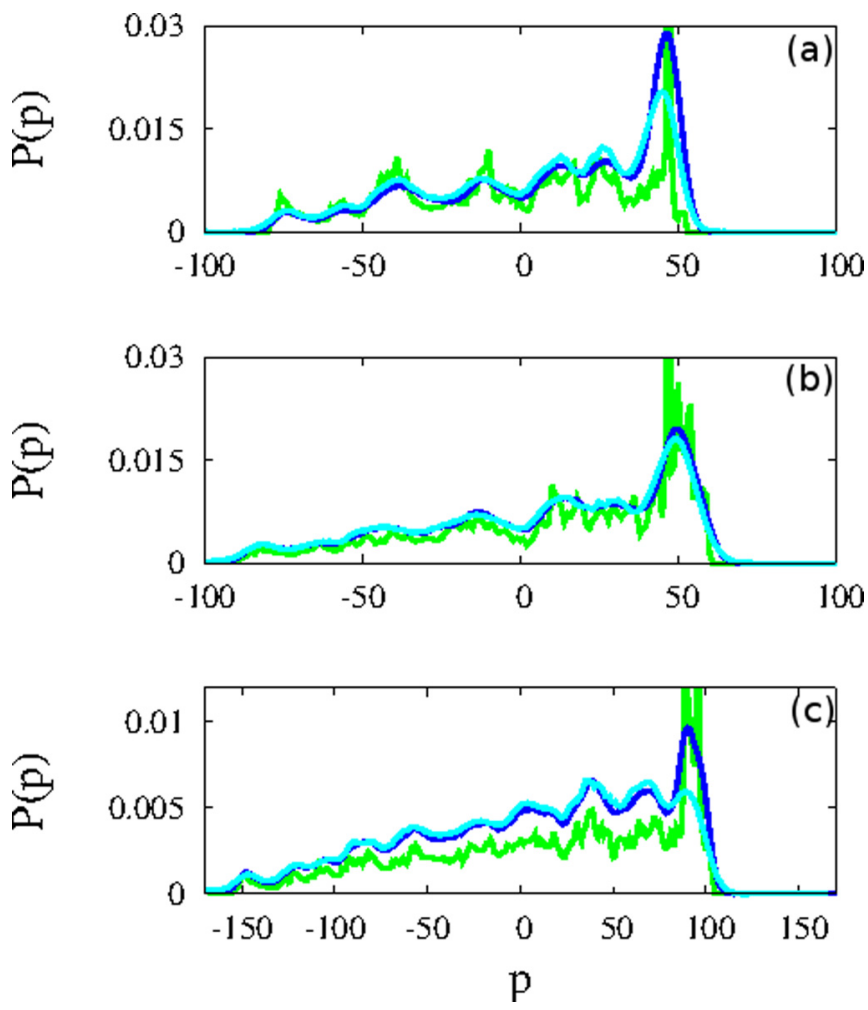

FIG. 5. $P(p)$ probability distributions as a function of $p$ for the three cases shown in Fig. 4, in the same order. Blue (black) lines correspond to the classical distributions with thermal noise, green (gray) lines to the ones without thermal noise, and cyan (light gray) lines to the quantum distributions.

performance is good. In the left column we display the classical distributions, and in the right one the quantum distributions. In Fig. 5 we show the probability distributions $P(p)$ as a function of $p$ that arise from the classical and quantum evolutions, in the same order as in Fig. 4.

The first example with $k=5$ and $\gamma=0.5$ belongs to a $B_{1}$ isoperiodic structure that is located in the red area of the largest dark region in Fig. 1(a), which has the greatest differences in the current values (the maximum being around 1.3, and in this case being of 0.74 ). The overlap between both phasespace distributions is $O=0.88$, which is good, but if we look at Fig. 5(a) we can see that there is an excess of classical probability around the location of the limit cycle associated with this structure (see the green line corresponding to the classical distribution without noise). This suggests that there should be a probability rebalancing due to purely quantum effects that takes it from this peak and transfers it to the chaotic basin which is next to it.

The second example with $k=5.55$ and $\gamma=0.55$ also belongs to $B_{1}$ but in this case it is located in a yellow area of this region in Fig. 1(a), reflecting the fact that the current behavior is quite similar. The overlap $O=0.94$ agrees with the difference in $J$ being just 0.13 , and the $P(p)$ distributions are almost the same [Fig. 5(b)]. If we compare both situations the main difference is the location of them in parameter space: while the first case is in what we would call the bulk of $B_{1}$ region the second is clearly at its border. This is the 
largest regular region and the influence of the surrounding areas of (mainly) chaos is reduced in its bulk. It seems that quantum effects enhance the probability transfer between these otherwise separated structures, going further than the rebalancing introduced by a plain coarse graining due to the size of $\hbar_{\text {eff }}$. As a matter of fact, we can clearly see the quantum nature of the distributions by means of the interference fringes that reveal the persistence of coherences despite the interaction with a quantum dissipative environment (at least at these finite values of the effective Planck constant).

Finally, the third example with $k=9.25$ and $\gamma=0.55$ belongs to the $B_{2}$ structure and is located in the second largest dark region in Fig. 1(a). The overlap is now $O=0.86$ which represents a performance comparable to the first case, with a difference in the current values of 0.73 . When looking at Fig. 5(c), the $P(p)$ distributions are also of the same nature as those of the first example. The behavior is exactly the same as for $B_{1}$, so our explanation of these discrepancies is of a general nature.

\section{CONCLUSIONS}

We have systematically studied the classical-quantum correspondence via thermal noise in a paradigmatic directed transport system, the dissipative modified kicked rotator. We have explored a good portion of the parameter space in terms of the asymptotic currents and the spectra of both the classical operators and the quantum superoperators. The first approach, though confirming the overall correspondence, gave us the expected picture of a nonuniform shape, dependent on the kind of dynamics underlying each region. On the contrary, the spectral study resulted in a uniform behavior. By focusing on the morphology of the equilibrium eigenstates, we could verify through well-developed interference fringes that the quantum nature is persistent at this finite $\hbar_{\mathrm{eff}}$, despite the environmental effects. This resilient quantumness is responsible for an enhancement of probability transfer from the main regular structures to the ones surrounding them in parameter space.

An effective (semi)classical map with noise has been proposed as a direct replacement for the quantum system and some results have been derived from this identification [22]. This map is quite similar to our much simpler approach, which is just a map with thermal noise with no free parameters. Ours is based on the general assumption that the main features of the quantum coarse graining are similar to those of a Gaussian coarse graining with size of the order of $\hbar_{\mathrm{eff}}$, in this directed transport system. This can be considered simplistic but it turns out to be precise enough in the overwhelming majority of the cases.

Of course, there are known discrepancies [18,20]. Again, we ascribe the difference in the asymptotic currents for some specific cases to finite $\hbar_{\text {eff }}$ quantum effects. In [32] it has been shown that for dissipative Markovian systems, with a generic Hamiltonian but linear dissipative Lindblad operators, in the semiclassical limit, the Wigner function evolves into a positive-definite phase-space distribution. However, in Fig. 4 the Wigner functions of the equilibrium eigenstates display negative values due to interference fringes. This shows that for the value of $\hbar_{\text {eff }}$ used here, we have not reached the semiclassical limit. As such, we conclude that though the classical dynamics with Gaussian, thermal noise is a very good approximation to the quantum dynamics, quantum effects could still be non-negligible, making a direct replacement an oversimplification. In fact, if one wants to develop a semiclassical approximation of theoretical interest and amenable to the vast majority of experimental situations in the quantum realm, coherences are needed. This idea is still under investigation and will be the subject of future works.

\section{ACKNOWLEDGMENT}

Support from CONICET under Project PIP 112201101 00703 is gratefully acknowledged.
[1] R. P. Feynman, Lectures on Physics (Addison-Wesley, Reading, MA, 1963), Vol. 1.

[2] P. Reimann, Phys. Rep. 361, 57 (2002).

[3] S. Kohler, J. Lehmann, and P. Hänggi, Phys. Rep. 406, 379 (2005).

[4] S. Denisov, S. Flach, and P. Hänggi, Phys. Rep. 538, 77 (2014).

[5] F. Jülicher, A. Ajdari, and J. Prost, Rev. Mod. Phys. 69, 1269 (1997); G. Mahmud et al., Nat. Phys. 5, 606 (2009); G. Lambert, D. Liao, and R. H. Austin, Phys. Rev. Lett. 104, 168102 (2010).

[6] R. D. Astumian, Science 276, 917 (1997); D. Reguera, A. Luque, P. S. Burada, G. Schmid, J. M. Rubí, and P. Hänggi, Phys. Rev. Lett. 108, 020604 (2012).

[7] J. B. Gong and P. Brumer, Annu. Rev. Phys. Chem. 56, 1 (2005); G. G. Carlo, L. Ermann, F. Borondo, and R. M. Benito, Phys. Rev. E 83, 011103 (2011).

[8] P. H. Jones, M. Goonasekera, D. R. Meacher, T. Jonckheere, and T. S. Monteiro, Phys. Rev. Lett. 98, 073002 (2007); T. Salger, S. Kling, T. Hecking, C. Geckeler, L. Morales-Molina, and M. Weitz, Science 326, 1241 (2009).
[9] T. S. Monteiro, P. A. Dando, N. A. C. Hutchings, and M. R. Isherwood, Phys. Rev. Lett. 89, 194102 (2002); G. G. Carlo, G. Benenti, G. Casati, S. Wimberger, O. Morsch, R. Mannella, and E. Arimondo, Phys. Rev. A 74, 033617 (2006).

[10] M. Sadgrove, M. Horikoshi, T. Sekimura, and K. Nakagawa, Phys. Rev. Lett. 99, 043002 (2007); I. Dana, V. Ramareddy, I. Talukdar, and G. S. Summy, ibid. 100, 024103 (2008).

[11] E. Lundh and M. Wallin, Phys. Rev. Lett. 94, 110603 (2005); D. Poletti, G. G. Carlo, and B. Li, Phys. Rev. E 75, 011102 (2007).

[12] A. Kenfack, J. Gong, and A. K. Pattanayak, Phys. Rev. Lett. 100, 044104 (2008); J. Wang and J. Gong, Phys. Rev. E 78, 036219 (2008).

[13] M. Sadgrove, M. Horikoshi, T. Sekimura, and K. Nakagawa, Eur. Phys. J. D 45, 229 (2007).

[14] S. Flach, O. Yevtushenko, and Y. Zolotaryuk, Phys. Rev. Lett. 84, 2358 (2000).

[15] J. L. Mateos, Phys. Rev. Lett. 84, 258 (2000).

[16] G. G. Carlo, G. Benenti, G. Casati, and D. L. Shepelyansky, Phys. Rev. Lett. 94, 164101 (2005). 
[17] A. Celestino, C. Manchein, H. A. Albuquerque, and M. W. Beims, Phys. Rev. Lett. 106, 234101 (2011).

[18] G. G. Carlo, Phys. Rev. Lett. 108, 210605 (2012).

[19] L. Ermann and G. G. Carlo, Phys. Rev. E 91, 010903(R) (2015).

[20] G. G. Carlo, A. M. F. Rivas, and M. E. Spina, Phys. Rev. E 92, 052907 (2015).

[21] J. Kullig and J. Wiersig, New J. Phys. 18, 015005 (2016).

[22] M. W. Beims, M. Schlesinger, C. Manchein, A. Celestino, A. Pernice, and W. T. Strunz, Phys. Rev. E 91, 052908 (2015).

[23] G. Lindblad, Commun. Math. Phys. 48, 119 (1976).

[24] T. Dittrich and R. Graham, Europhys. Lett. 7, 287 (1988).

[25] R. Graham, Z. Phys. B Condens. Matter 59, 75 (1985).
[26] S. M. Ulam, A Collection of Mathematical Problems, Interscience Tracts in Pure and Applied Mathematics (Interscience, New York, 1960).

[27] L. Ermann and D. L. Shepelyansky, Eur. Phys. J. B 75, 299 (2010); K. M. Frahm and D. L. Shepelyansky, ibid. 76, 57 (2010).

[28] L. Ermann, K. M. Frahm, and D. L. Shepelyansky, Rev. Mod Phys. 87, 1261 (2015).

[29] G. W. Stewart, Matrix Algorithms Vol. II: Eigensystems (SIAM, Philadelphia, PA, 2001).

[30] A. M. F. Rivas and A. M. Ozorio de Almeida, Ann. Phys. (NY) 276, 223 (1999).

[31] A. Argüelles and T. Dittrich, Physica A 356, 72 (2005).

[32] A. M. Ozorio de Almeida, P. de M. Rios, and O. Brodier, J. Phys. A: Math. Theor. 42, 065306 (2009). 